\title{
Chaos theory and applications: a retrospective on lessons learned and missed or new opportunities
}

\author{
Laura Gardini · Celso Grebogi · Stefano Lenci
}

Published online: 27 August 2020

(C) Springer Nature B.V. 2020

Chaos, from the Greek khaos, "abyss, that which gapes wide open, that which is vast and empty", is a relatively young scientific discipline, which however has old and important roots, dating back (at least) to James Clerk Maxwell in 1860 and Henry Poincaré in 1890. The birth of the modern age of Chaos is commonly referred to the work of Edward Lorenz in 1963. After this triggering point, it became a flourishing and fascinating research field that attracted the interest of top scientists as well as many valuable researchers, still active to date. Looking for the word "Chaos" in modern research tools, an almost uncountable number of papers appear.

After 60 years of huge developments, after books (J. Gleick, Chaos; R. Abraham and Y. Ueda, The Chaos Avant-Garde: Memories of the Early Days of Chaos Theory; etc.) and Symposia (IUTAM Symposium on 50 Years of Chaos: Applied and Theoretical, Kyoto, 2011, etc.), we felt it was the right time for a pitstop, aimed at looking:

\footnotetext{
L. Gardini

Urbino, Italy

C. Grebogi

Aberdeen, UK

S. Lenci $(\bowtie)$

Ancona, Italy

e-mail: lenci@univpm.it
}

1. backward, of course, to "summarize" the major developments and their applications, successes and failures;

2. forward, to see the new ongoing developments of this fascinating discipline and future perspectives, in particular unexplored ones; and

3. laterally, i.e. to works that while strictly not classifiable as belonging to the chaos family, are close and relevant indeed, having common features.

We have also tried to give an answer that is necessarily partial and incomplete, to the questions "Who were we?", "Who are we?", "Who will we be?" in Chaos.

According to this vision, we selected and invited a limited number of top scientists to contribute to this special issue, asking them to bring their ideas, reflections, viewpoints, developments, regrets, etc. Thus, the special issue was open to review papers, to position papers as well as, of course, to technical papers, even those not specifically referring to Chaos. The enthusiastic responses of the invited scholars have been encouraging.

Chaos is intrinsically multidisciplinary, and it is of interest and find applications in mathematics, science, engineering, physics, economics, chemistry, biology, medicine, etc. Without exaggeration, we can say that almost any system that evolves in time (dynamical system) is susceptible to be chaotic, whatever its meaning, its evolution equations, its dimension, etc. 
The Guest-Editor composition reflects, as much as possible, this fundamental aspect: Gardini, from mathematics and economy, Grebogi, from physics, and Lenci, from engineering. Also, the invited authors have been selected with the aim of representing and typifying the interdisciplinarity of Chaos.

One of the most famous property of Chaos is the sensitivity to the initial conditions, also popularly known as "Butterfly effect" after Lorenz in 1972 (but although known to Maxwell and Poincaré) and after movies like "Sliding doors". It entails, even if the system is totally deterministic and known, that small changes in initial conditions lead, sooner or later, to large differences. In other words, this means that in the chaotic realm, we are not able to make long-term predictions, unless we are able to know exactly the starting point, which is of course not the case in the real world. According to this distinct property, we cannot predict whether this special issue will be relevant or not. We can only hope that it will be of interest for both senior scholars, aimed at knowing a little bit more about Chaos, and younger researchers, aimed at finding new ways, new results and new applications.

We wish to thank all the authors for their hard work, and for supporting our idea. Last but not least, we wish to thank the Editor-in-Chief of Nonlinear Dynamics, Prof. Walter Lacarbonara that not only hosts this special issue, but triggered it and helped during its development.

Publisher's Note Springer Nature remains neutral with regard to jurisdictional claims in published maps and institutional affiliations. 